\title{
Scheduling Preventive Railway Maintenance Activities
}

\author{
Gabriella Budai, Dennis Huisman and Rommert Dekker \\ Econometric Institute, Erasmus University Rotterdam \\ P.O. Box 1738, 3000 DR, Rotterdam, the Netherlands \\ E-mail: \{budai, huisman, rdekker\}@few.eur.nl
}

September 15, 2004

\section{Econometric Institute Report EI 2004-41}

\begin{abstract}
A railway system needs a substantial amount of maintenance. To prevent unexpected breakdowns as much as possible, preventive maintenance is required. In this paper we discuss the Preventive Maintenance Scheduling Problem (PMSP), where (short) routine activities and (long) unique projects have to be scheduled in a certain period. To reduce costs and inconvenience for the travellers and operators, these activities have to be scheduled as much as possible together. We present a mathematical formulation for this problem and some greedy heuristics to solve it fast. Moreover, we compare the performance of these heuristics with the optimal solution using some randomly generated instances.
\end{abstract}

Keywords: Rail transport, Maintenance, Scheduling, Optimization, Heuristics

\section{Introduction}

Reliability, i.e. punctuality and safety, are important aspects in railway transport. The quality of the railway infrastructure has a major influence on the reliability of the railway system as a whole. Therefore, it is important that there is enough preventive maintenance of the infrastructure (e.g. rail, ballast, sleepers, switches and fasteners). However, maintenance is very expensive and budgets for maintenance are always under pressure. For instance, the 
Dutch government drastically reduced the amount of money spent to maintenance at the end of the nineties with major consequences on the punctuality of the railway system a couple of years later. So it is important to reduce the maintenance costs without reducing the maintenance itself. One of the aspects which will be considered in this paper, is to combine maintenance activities on the same link in the network as much as possible. Then, the railway traffic is disturbed as less as possible and the costs for taking a track out of service are incurred only once.

In this paper, we consider the Preventive Maintenance Scheduling Problem (PMSP), where a schedule for the maintenance activities has to be found for one link such that the possession costs are minimized. These possession costs are mainly determined by the possession time, which is the time that a track is required for maintenance. In that case, it is blocked for train traffic and handed over by the railway operators to the maintenance engineers, who take "possession" of the track. When the track is returned to the operators, the engineers "give up possession".

In this paper, we focus on the medium (long) term planning, determining which preventive maintenance works will be performed on which segments in which time periods (month/week/hours), minimizing the track possession cost (which is mostly equal to the track possession time). The contribution of this paper is twofold. First, we will provide a mathematical formulation for the PMSP. Second, we will give some heuristics to solve this problem fast. The latter is important, since in practice a whole network needs to be optimized. In the next section we explain how this can be done using the concept of single track grids suggested by Van Zante-de Fokkert et al. (1). Moreover, to give an insight in the quality of the heuristics, we will compare them with (a lower bound on) the optimal solution.

The remainder of the paper is organized as follows. In next section we place our paper in the railway world and the existing literature on maintenance scheduling of railway infrastructure. A formal problem description is given subsequently, followed by a mathematical formulation. After that we present some heuristics to solve the problem approximately. Finally, we conclude the paper with some computational experiments.

\section{Railway maintenance planning}

Preventive railway maintenance works are performed in order to reduce the probability of the occurrence of a failure on the components of the railway infrastructure and/or maximise the operational benefit (see Kumar et al. (2)). The frequency of these works may be based on calender time, operating 
time or depending on the actual condition of the infrastructure components.

Preventive maintenance on railways can be subdivided into small routine works and projects. The routine (spot) maintenance activities consist of inspections or small repairs, e.g. inspection of rail, switch, level crossing, overhead wire, signalling system and switch lubrication (see Esveld (3)) These works do not take much time to be performed and are done frequently, few times a year. The projects includes renewal works and consists e.g. of ballast cleaning, rail grinding and tamping (see Esveld (3)). They are carried out once/twice in a few years.

Budai \& Dekker (4) and Improverail (5) shows that the preventive railway maintenance works are carried out in most countries during train service. In the actual train timetable possible possession allocations are scheduled for maintenance so that it should not affect too much the regular train service. Many countries use timetabling software (e.g. Viriato (6), Opentrack (7)) for finding free intervals or periods with less impact to the train operators. Carrying out maintenance works during train services might be unsafe for the maintenance crew. Therefore, in some countries the maintenance works should be carried out either night (when there are only few trains) or during day with interruption of the train service. Because of the safety requirements train cancellation is required, so one has to arrange alternative transport (e.g. using buses) during the track possession time. In Higgins (8) and Cheung et al. (9) the track possession is modelled in between operations. As we have already explained, this can be done for occasionally used tracks or if the maintenance crew accepts the risk of working on the track during train service. If tracks are used frequently or the risks are not acceptable, then the maintenance works have to be performed during day, blocking the train operation or during nights, when the train traffic is almost absent. In the latter case one can either make a cyclic static schedule, which is made by Den Hertog et al. (10) and Van Zante-de Fokkert et al. (1) for the Dutch situation or a dynamic schedule with a rolling horizon, which is presented in Cheung et al. (9). Miwa et al. (11) presents an optimal schedule for a specific maintenance work, namely an annual schedule for the tie (sleeper) tamping. In this paper we assume that the risk of maintaining the tracks during the train services is not acceptable. Therefore every time when a track is maintained, it is blocked for the train service. Furthermore, in these track possessions the maintenance activities (routine works and projects) are clustered as much as possible, since then the railway operation is disturbed as little as possible.

As it has been mentioned in the previous section, the maintenance activities are scheduled in our problem on each link separately. However, in practice the optimization is done for the whole network, taking into account 
the geographical position of the track links. Van Zante-de Fokkert et al. (1) suggests a method for defining Single Track Grids (STGs). An STG covers a set of sections on a particular corridor that can be out of service at the same moment without disrupting the railway traffic too severely. The STGs on the same corridor or sometimes STGs on different corridors cannot be combined simultaneously because it would result in too much disturbances for the train operation. So, using the concept of Van Zante-de Fokkert et al. (1) in PMSP a link can be understood as a group of links (i.e. an STG) which can be taken out of service at the same time. This means actually, that the maintenance works on several links will be scheduled together instead of scheduling the works from each link separately.

Solving the preventive railway maintenance scheduling problem to optimality is a very difficult and complex problem, since usually the models contain many integer variables. Sometimes a fast computation time is necessary. Therefore, some heuristics have been developed. In Higgins (8) the tabu search heuristic technique was used to find a solution to the large 0-1 integer program. Another way for planning railway track maintenance works is by using Genetic Algorithm and Genetic Programming methods, which is presented in Grimes (12). This procedure was shown to perform well for a very small number of samples and quite bad for bigger instances.

\section{Problem description}

In this paper our aim is to give a schedule for preventive maintenance activities, such that jobs are clustered as much as possible in the same period. Recall that we only consider the problem per link, but it can be generalized to multiple links using the concept of STGs.

The PMSP can be defined as follows. Given a set of routine activities and projects, we like to schedule them such that the track possession costs are minimized. These costs are mostly proportional to the track possession times, which means that the preventive maintenance works should be clustered as much as possible. For each routine work we know the number of time periods between two consecutive executions, i.e. the frequency of such a work can be calculated. Furthermore, we know the duration and the earliest and latest possible starting times of each project, which needs to be performed in the planning period. Finally, we know which routine works can be combined with each other and with which projects. We assume that projects cannot be combined, since it would take too much time and manpower.

At first sight the model presented in this paper seems to be related to the machine scheduling problem and the multi-project scheduling problem in an 
abstract way. The similarity among these three problems is that there are some jobs, with given durations and given time windows between two consecutive executions, which have to be scheduled in a certain time period. One of the differences is that in PMSP the routine works have predefined frequencies, i.e. they have a cyclic pattern. Furthermore, the objective of PMSP is different than the objectives of the other mentioned problems. Namely, we want to schedule the jobs as much as possible together and not necessarily as soon as possible. Since in our problem beside the routine works (with cyclic pattern) some projects are also scheduled and some works can be and some other cannot be combined, we can conclude that the PMSP has a different structure than these two above mentioned problems.

\section{Mathematical Formulation}

Let $T$ be a set of discrete time periods (e.g. months, weeks) in which the maintenance activities need to be scheduled, i.e. $|T|$ is the planning horizon. Furthermore, define $A=P A \cup R A$ as the set of all activities, which includes a set of projects $(P A)$ and a set of routine maintenance works $(R A)$. Moreover, define $C$ as the set of combinable works, i.e. $C=\{(m, n) \mid$ work $\mathrm{m}$ is combinable with n, $\forall m, n \in A\}$. For each routine maintenance work $a \in R A$, the cycle length $L^{a}$ and its corresponding frequency $F^{a}=\left\lfloor\frac{|T|}{L^{a}}\right\rfloor$ is given. For the projects a set of possible start points $T_{p} \in T$ is known for each project $p$. Moreover, each project $p \in P A$ has a duration $D_{p}$. Finally, we define $c_{t}$ as the possession cost, i.e. cost for carrying out maintenance in period $t \in T$.

The following binary decision variables are defined:

$x_{t}^{a} \quad$ binary variable that denotes whether activity $a \in A$ is assigned to period $t \in T\left(x_{t}^{a}=1\right)$, or not $\left(x_{t}^{a}=0\right)$,

$m_{t} \quad$ binary variable that denotes whether this link is used for preventive maintenance work at time $t \in T\left(m_{t}=1\right)$, or not $\left(m_{t}=0\right)$,

$y_{t}^{p} \quad$ binary variable that denotes whether the execution of project $p \in P A$ starts at time $t \in T\left(y_{t}^{p}=1\right)$, or not $\left(y_{t}^{p}=0\right)$.

The Preventive Maintenance Scheduling Problem can now be formulated as follows:

$$
(P M S P) \quad \operatorname{Min} \sum_{t \in T} c_{t} m_{t}
$$


s.t.

$$
\begin{array}{cc}
\sum_{t=1}^{L^{a}} x_{t}^{a}=1 & \forall a \in R A \\
x_{t}^{a}=x_{t+q \cdot L^{a}}^{a} & \forall a \in R A, t=\left\{1, \ldots, L^{a}\right\}, 1 \leq q \leq F^{a}-1 \\
x_{t}^{m}+x_{t}^{n} \leq 1 & \forall t \in T,(m, n) \notin C \\
\sum_{t \in T_{p}} y_{t}^{p}=1 & \forall p \in P A \\
x_{s}^{p} \geq y_{t}^{p} & \forall p \in P A, t \in T_{p}, s=t, \ldots, t+D_{p}-1 \\
m_{t} \geq x_{t}^{a} & \forall a \in A, t \in T \\
x_{t}^{a}, y_{t}^{p}, m_{t} \in\{0,1\} & \forall a \in A, p \in P A, t \in T,
\end{array}
$$

The objective minimizes the possession costs for the time periods for which maintenance work is planned. Actually, if $c_{t}=1 \forall t \in T$ the objective function minimizes the track possession time for routine maintenance works and projects. Constraints (2) ensure that each routine maintenance work is scheduled exactly once in the first planning cycle and then constraints (3) guarantee that until the end of the planning horizon the works for the other cycles will be defined as well, ensuring $L^{a}$ time periods between two subsequent occurrences of the same job. On the same link and at the same time only combinable activities can be carried out. This is ensured by constraints (4). These combinable jobs can be either routine works or projects. Constraints (5) guarantee that each project is executed once. Furthermore, constraints (6) ensure that each project is assigned to the right number of time periods and the starting time for performing the projects is in the interval (earliest possible starting time, latest possible starting time). Furthermore, these projects are assigned to subsequent intervals. Constraints (7) ensure that time period $t \in T$ will be occupied for preventive maintenance work if and only if for that time period on this segment at least one work is planned. Finally, constraints (8) ensure that the decision variables are binary.

\section{Solution approach}

The PMSP is modelled in GAMS and it is solved afterwards with the MIP solver CPlex 7.1. Solving this optimization problem to optimality for a single link, more than 15 types of maintenance works and for more than 3-4 years, requires a huge amount of time. It is desirable to compute the optimal solution, but since it takes too long time to find this solution it might be better to settle for a non-optimal solution which has somewhat larger track 
possession cost, but is still quite close to the optimal objective value, and which can be found in a reasonable time. Therefore, we develop three simple, greedy heuristics for solving the PMSP. In "Simple Heuristics" each of them is presented in detail. It is worth to mention that in each of the approximation methods we schedule the routine maintenance works and projects together. If two or more routine works cannot be combined then they will be scheduled for separate time periods. Otherwise they might be scheduled and carried out at the same time. Before we discuss the heuristics, we discuss some tricks to reduce the problem size.

\section{Problem reduction techniques}

If two activities have the same planning cycle then we could combine them into a "super" activity and do the planning for that one. This idea to reduce the problem size can be extended as it is formulated in the following theorem.

Theorem 1 Let $c_{t}=1 \forall t \in T$. Furthermore, let $a$ and $b, a, b \in R A$ be two different types of preventive maintenance activities which can be scheduled at the same time and let $L^{a}$ and $L^{b}$ be the planning cycles and $F^{a}$ and $F^{b}$ be the frequency of these works, where $L^{a} \geq L^{b}$. If

$$
\exists k \in \mathcal{N}: L^{a}=k \cdot L^{b}
$$

then the optimal value of the scheduling problem is equal to the optimal value of the LP relaxation of PMSP and they are both equal to $F^{b}$.

Proof. Let's assume that for a certain $t \in\left\{1, \ldots, L^{a}\right\}: x_{t}^{a}=1$. Then, $x_{t+(i-1) \cdot L^{a}}^{a}=1 \forall i \in\left\{1, \ldots, F^{a}\right\}$. Thus $1=x_{t+(i-1) \cdot L^{a}}^{a}=x_{t+(i-1) \cdot k \cdot L^{b}}^{a}=$ $x_{t+p \cdot L^{b}}^{b}=x_{p}^{b}$, where $p=(i-1) \cdot k, p, k \in \mathcal{N}$. This means that in each period work $a$ is scheduled, there is a work $b$ as well. Thus the optimal value is equal to $F^{b}$.

Since we minimize $\sum_{t \in T} m_{t}$ and constraints (7) guarantee that each $m_{t} \geq$ $x_{t}^{a}$, the value of the LP relaxation of PMSP is at least $\sum_{t \in T} x_{t}^{a}$, which is equal to $F^{b}$. Since we showed above that the optimal solution has value $F^{b}$, this is the value of the LP relaxation as well.

Using the following remark we can immediately check whether two or more works can be combined or not.

Remark 1 Let $a$ and $b, a, b \in R A$ be two different types of preventive maintenance activities which cannot be scheduled at the same time. Their frequencies and planning cycles are denoted by $F^{a}$ and $L^{a}$, and $F^{b}$ and $L^{b}$. If 


\section{$L^{a}, L^{b}$ are relatively prime and $l c m\left(L^{a}, L^{b}\right) \leq T$}

then there is no feasible solution for the maintenance work scheduling problem.

Although both statements deal with two activities, they can be obviously generalized to more than two activities. We will use them to reduce the problem size of the instances before solving the actual problem. Thus, if some of the preventive maintenance works are such that their planning cycles equals or are multiples of at least one work's planning cycle, then it is clever to group these works, having a common planning cycle for the whole group equal to the minimum of these works' cycles. Actually, in this way, only one representative work of this group will be scheduled, the one with the smallest planning cycle. The other works will be introduced in the schedule later. In this way, the number of works in the maintenance scheduling problem will be reduced, which reduces the problem size as well. Moreover, we can immediately check if there is an infeasible solution for an instance. In practice, this can be used to change the planning cycles of the maintenance activities.

\section{Simple Heuristics}

As we already mentioned before, the railway maintenance scheduling problem is a difficult optimization problem to solve in a short time. Therefore, we present three simple greedy heuristics. They are greedy in sense that they try to combine every activity together. The first heuristic starts with scheduling the works having the highest frequency, the second heuristic with the works having the lowest frequency and the third heuristic, which is the simplest from these three heuristics, schedules every work for the first time period. Usually the greedy heuristic does not give very good results, therefore we included some local improvements steps in the first two heuristics.

Before we describe the heuristics, we recall the input of the problem and some notation. It is given a set of routine maintenance works $R A$ with their planning cycle $L^{a}$ and frequencies $F^{a}, \forall a \in R A$ and a set of projects $P A$ with duration $D_{p}$ and a set of possible start points $T_{p} \in T$. There is also a list of works which cannot be scheduled together.

\section{Max to Min heuristic (MaMi)}

Step 0

- Order the set of routine works $R A=\{1, \ldots, n\}$ such that the frequencies are in decreasing order, i.e. $F^{1} \geq F^{2} \geq \ldots \geq F^{n}$. 
- Schedule routine work 1 in period $1,1+L^{a}, \ldots$ etc.

Step 1 For $\mathrm{j}=2, \ldots, \mathrm{n}$

- Schedule routine work $j$ such that the increase in track possession cost is minimal. Take into account that there are certain periods where work $a$ cannot be scheduled due to the earlier choices made for works $1, . ., j-1$.

Step 2

- Choose a project $p$ from set $P A$ with the earliest possible starting time. In the allowed time interval $T_{p}$ find the best time moment for performing it together as much as possible with already scheduled routine maintenance works.

Step 3

- Repeat Step 1 and 2 for all values where work 1 can be done the first time.

- The schedule resulting in the minimum track possession cost is chosen.

\section{Min to Max heuristic (MiMa)}

The difference between this heuristic and the previous one is that here we order the routine works such that the frequencies are in increasing order, i.e. we start with the one with the lowest frequency.

The last heuristic can be formulated in the following way:

\section{Combine Everything in the First Period (CEFP)}

- In period 1 each of these $n$ routine maintenance works will be scheduled. According to the works' frequency and planning cycle the rest of the time moments will be defined.

- The identified projects from set $P A$ will be scheduled in the following way: start performing the projects at their first possible starting time. If performing a project takes longer than one time period, then schedule it for consecutive time periods; 
- Check whether for the same time period two works has been scheduled which are actually forbidden to be carried out together. If not, then do not do anything. If yes, then shift the schedule of one of these works to the right until there are no overlapping time periods. If there are no more possibilities for shifting (the end of the first planning cycle has been reached) and until that moment was not possible to schedule these two works, then no feasible solution could be found, otherwise calculate the track possession cost.

As we already mentioned, all three heuristics are greedy heuristics containing some improvement steps. The complexity of these algorithms is as follows: MaMi and MiMa have complexity of $O\left(n(n+p) T^{2}\right)$ and CEFP $O((n+p) T)$, where $n$ is the number of routine maintenance works and $p$ is the number of projects.

\section{Computational results}

In this section computational results for some randomly generated instances are presented. The planning horizon for these instances is two years and the discrete time periods are weeks. Furthermore, we assume that each routine maintenance work has different planning cycles and consequently different frequencies. Here we assume, that $c_{t}=1 \forall t \in T$, so the objective function minimizes the track possession time for routine maintenance works and projects.

To test the algorithms, we generated instances with 15, 20 and 25 routine works. For each size we generated 10 instances. The generated values for the planning cycles are uniformly distributed random numbers in the interval $[4,52]$ and from the planning cycles one can calculate the frequency of each work. Moreover, we generated uniformly distributed random numbers in the interval $[0,2]$ for the number of projects which have to be performed until the end of the planning horizon for each separate set. The possible earliest and latest starting times are randomly generated values from the interval $[1,104]$. The duration of a project is also a uniformly distributed random number in the interval $[1,6]$.

In the preprocessing phase we checked the generated data and according to Theorem 1 we can make groups of routine maintenance works which have the same, or multiple length planning cycles. In this way the original 10 instances containing 25 works were reduced to 10-15 works, the instances containing 20 works reduced to $7-14$ works and the last 10 instances have after the reduction 6-13 works. Furthermore, we tested our model for two 


\begin{tabular}{|c|c|c|c|c|c|c|}
\hline & \multicolumn{2}{|c|}{$n=25$} & \multicolumn{2}{|c|}{$n=20$} & \multicolumn{2}{|c|}{$n=15$} \\
\hline & Average & St.dev & Average & St.dev & Average & St.dev \\
\hline \multicolumn{7}{|c|}{ Scenario1: Combination of works is allowed } \\
\hline SFre & 82.3 & 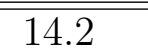 & 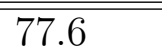 & 19.5 & 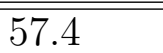 & 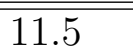 \\
\hline $\mathrm{NC}$ & 2376.5 & 370.8 & 2015.8 & 454.3 & 1763.2 & 404.3 \\
\hline NV & 1522.8 & 222.7 & 1306.5 & 250.5 & 1165 & 264.6 \\
\hline VOpt & 42.6 & 7.22 & 42.4 & 7.74 & 33.4 & 7.96 \\
\hline TOpt(sec) & 3454.1 & 5669.7 & 6871.6 & 17447.8 & 3753.6 & 9378.4 \\
\hline VLP & 19.8 & 4.9 & 21.9 & 4.5 & 18.4 & 4.8 \\
\hline $\operatorname{TLP}(\mathrm{sec})$ & 0.37 & 0.35 & 0.2 & 0.1 & 0.2 & 0.1 \\
\hline \multicolumn{7}{|c|}{ Scenario2: Some works cannot be combined } \\
\hline SumFreq & 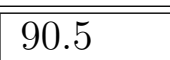 & 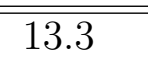 & 84.4 & 19.1 & 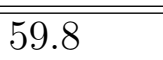 & $\overline{12.2}$ \\
\hline NoCon & 3195.9 & 306.3 & 2772.2 & 363.8 & 2352.8 & 371.2 \\
\hline NoVar & 1762 & 187.9 & 1524.9 & 199.1 & 1284.2 & 231.8 \\
\hline VOpt & 44.1 & 8.6 & 43.7 & 8.5 & 34.1 & 8.1 \\
\hline TOpt(sec) & 6132.8 & 11718 & 3185.5 & 4998.7 & 4114.6 & 6610 \\
\hline VLP & 19.8 & 4.9 & 21.9 & 4.5 & 16.3 & 5.6 \\
\hline $\operatorname{TLP}(\sec )$ & 0.5 & 0.3 & 0.4 & 0.4 & 0.4 & 0.4 \\
\hline
\end{tabular}

Table 1: Problem specifications and Computational results

scenarios. In the first scenario we assume that each routine work can be combined with all other routine works and projects, but the projects cannot be combined with other projects. In the second scenario we assume that a group of 2 works and a group of three works cannot be combined. These works are arbitrary chosen. From the previous section it is known that if the planning cycles of two (or more) not combinable works satisfy the assumptions of Remark 1 then there is no feasible solution. To prevent this situation, we choose from each generated set two works and three works respectively, such that even if they are not scheduled together, we will get a feasible solution.

The results, after running the model for the generated instances, are shown in Table 1.

In Table 1 we first listed for all problem sizes the mean of the sum of the works' frequencies (SFre), the average number of variables (NV) and the average number of constraints (NC). Furthermore, the average optimal value (VOpt) is shown, i.e. the track possession time for routine works and projects and the standard deviation for the three cases separately $(n=25$, 20 and 15 , where $n=$ number of routine maintenance works). Moreover the average CPU time (TOpt) for finding the optimal solution, the average LP 


\begin{tabular}{|c|c|c|c|c|c|c|c|}
\hline \multirow[b]{2}{*}{ Heur. } & \multirow[b]{2}{*}{ Variable } & \multicolumn{2}{|c|}{$n=25$} & \multicolumn{2}{|c|}{$\overline{n=20}$} & \multicolumn{2}{|c|}{$n=15$} \\
\hline & & Average & St.dev & Average & St.dev & Average & St.dev \\
\hline \multicolumn{8}{|c|}{ Scenario1: Combination of works is allowed } \\
\hline \multirow{4}{*}{ MaMi } & "ObjV & "44.4 & 6.8 & "44.3 & 8.2 & 34.5 & 7.9 \\
\hline & ObjT(hsc) & 2.8 & 2.2 & 2.2 & 2.1 & 3.3 & 4.0 \\
\hline & VStp2 & 45.3 & 6.6 & 45.7 & 8.4 & 35.6 & 7.8 \\
\hline & $\mathrm{RDOH}(\%)$ & 4.2 & - & 4.4 & - & 2.9 & - \\
\hline \multirow{4}{*}{ MiMa } & ObjV & 45.7 & 6.9 & 44.6 & 9.0 & 35.6 & 7.3 \\
\hline & ObjT(hsc) & 19.6 & 2.3 & 17 & 3.1 & 17.1 & 2.5 \\
\hline & VStp2 & 50.4 & 7.1 & 49.6 & 9.8 & 39.3 & 7.4 \\
\hline & $\mathrm{RDOH}(\%)$ & 7.2 & - & 5.1 & - & 6.5 & - \\
\hline \multirow{3}{*}{ CEFP } & ObjV & 55.8 & 7.7 & 53.9 & 9.3 & 42.4 & 8.4 \\
\hline & ObjT(hsc) & 2.1 & 0.8 & 1.8 & 0.6 & 1.9 & 1 \\
\hline & $\mathrm{RDOH}(\%)$ & 30.9 & & 27.1 & & 26.9 & \\
\hline \multicolumn{8}{|c|}{ Scenario2: Some works cannot be combined } \\
\hline \multirow{4}{*}{ MaMi } & OObjV & 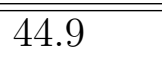 & 8.5 & 44.8 & 8.9 & 35.2 & 8.1 \\
\hline & ObjT(hsc) & 8.8 & 11 & 6.3 & 7.8 & 9.5 & 21.6 \\
\hline & VStp2 & 46.9 & 9.1 & 46.8 & 8.8 & 36.8 & 7.8 \\
\hline & $\mathrm{RDOH}(\%)$ & 1.8 & - & 2.5 & - & 3.2 & - \\
\hline \multirow{4}{*}{ MiMa } & ObjV & 47.5 & 9 & 47.6 & 9.7 & 37 & 7.8 \\
\hline & ObjT(hsc) & 158.5 & 90.5 & 122.2 & 79.8 & 120.5 & 83.8 \\
\hline & VStp2 & 53.6 & 7.6 & 51.6 & 10.3 & 41.6 & 8.4 \\
\hline & $\mathrm{RDOH}(\%)$ & 7.7 & - & 8.9 & - & 8.5 & - \\
\hline \multicolumn{2}{|c|}{ CEFP ObjV } & - & - & - & - & - & - \\
\hline
\end{tabular}

Table 2: Results for the three heuristics

relaxation value (VLP) and the average CPU time needed to find the LP relaxation value (TLP) is listed in this table separately for each scenario. The above mentioned results are listed for each scenario separately. All the tests are executed on a Pentium IV $1.60 \mathrm{GHz}$ (256MB RAM) personal computer, using CPLEX 7.1 for calculating the LP and the optimal integer solution.

In Table 2, one can find the mean of the heuristic solutions $(\mathrm{Obj} \mathrm{V})$ and the CPU times in hundredths of seconds (ObjT) for the three heuristics separately. For the first two heuristics the VStp2 value is shown, i.e. the objective value after Step2. From this value and the mean heuristic solution value we can understand how important it is to use Step 3 in the algorithm. We recall, that by Step 3 we try to improve the actual value of the heuristic, by shifting the first work's schedule to the left (until the end of the first 
planning cycle). The relative differences between the optimal value and the heuristic value $(\mathrm{RDOH})$ are given for each instance and for both scenarios.

From the results we can conclude that if we compare the optimal value and the value of the solution provided by the heuristics, then the first and the second heuristic perform well, since the heuristic values are very close to the optimal value. In the case of the first heuristic the solution is found right away, but for the second and third heuristics less than two seconds are needed for calculating a feasible solution. In comparison with the CPU time for calculating the optimal solution, the second heuristic is also very fast. The third heuristic gives quite poor solutions, the relative difference between the optimal value and the heuristic value is above $26 \%$.

Concluding, we can say that the Max to Min heuristic gives the best results compared to the other heuristics.

\section{Conclusions}

Since rail is an important transportation mode, proper maintenance of the existing lines, repairs and replacements carried out in time are all important to ensure efficient operation. Moreover, since some failures might have a strong impact on the safety of the passengers, it is important to prevent these failures by carrying out in time and according to some predefined schedules preventive maintenance works. Since the infrastructure maintenance costs represent a huge part of the total operating costs, there is a need for developing operations research tools, which help the maintenance planners to come up with optimal maintenance plans.

In this paper we present an optimization model to improve rail maintenance decisions by creating a schedule for carrying out preventive maintenance activities. Maintenance works are assigned to different time periods (months/weeks), minimizing the track possession cost or the track possession time. Routine maintenance works and projects are planned together. Furthermore, since the maintenance scheduling problem is a complex optimization problem and for a large set of instances it is difficult and time consuming to solve the problem to optimality, it is necessary to develop some approximation methods, which still give solutions close to the optimal ones.

From the three discussed heuristics it turned out that the Max to Min heuristic performs the best. This heuristic gives a 3-4\% worse solution than the optimal solution, but the running time is negligible.

As a final remark we like to mention that the model presented in this paper is just a basic model, but it can be extended to solve all types of practical problems, since in reality there are much more constraints that a 
maintenance planner has to take into account. One possible extension could be to schedule the maintenance work and crew together. Another example is the case of multiple operators with different time preferences (e.g the UK).

Acknowledgments We would like to thank the experts from the Strukton Railinfra, the Netherlands, for providing us information about planning and performing railway maintenance activities.

\section{References}

1 Van Zante-de Fokkert, J.I., Den Hertog, D., Van den Berg, F.J., \& Verhoeven, J.H.M. (2001). Safe Track Maintenance for the Dutch Railways, Part II: Maintenance schedule. Tech. rept. Tilburg University, the Netherlands.

2 Kumar, U. Dinesh, Crocker, J., Knezevic, J., \& El-Haram, M. (2000). Reliability, maintenance and logistic support - A life cycle approach. Kluwer Academic Publishers, Boston/Dordrecht/London.

3 Esveld, C. (2001). Modern Railway Track. MRT-Productions, Zaltbommel, The Netherlands.

4 Budai, G., \& R., Dekker. (2002). An overview of techniques used in planning railway infrastructure maintenance and its effect on capacity. In Geraerds, W.M.J., \& Sherwin, D. (eds), Proceeding of the IFRIMmmm (maintenance management and modelling conference), Växjö, Sweden.

5 Improverail. http://www.tis.pt/proj/improverail/Downloads/D6Final.pdf accessed 10 September 2004.

6 Viriato. http://www.sma-partner.ch accessed 10 September 2004.

7 Opentrack. http://www.opentrack.ch accessed 10 September 2004.

8 Higgins, A. (1998). Scheduling of railway maintenance activities and crews. J Opl Res Soc, 49, 1026-1033.

9 Cheung, B.S.N., Chow, K.P., Hui, L.C.K., \& Yong, A.M.K. (1999). Railway track possession assignment using constraint satisfaction. Engineering Applications of AI, 12(5), 599-611.

10 Den Hertog, D., Van Zante-de Fokkert, J.I., Sjamaar, S.A., \& Beusmans, R. (2001). Safe Track Maintenance for the Dutch Railways, Part I: Optimal working zone division. Tech. rept. Tilburg University, the Netherlands. 
11 Miwa, M., Ishikawa, T., \& Oyama, T. (2001). Modeling the optimal Decision-Making for Multiple Tie Tamper Operations. In Proceedings of the 5th World Congress on Railway Research, Cologne, Germany.

12 Grimes, C.A. (1995). Application of Genetic Techniques to the Planning of Railway Track Maintenance Work. In Zalzala, A.M.S. (ed), First International Conference on Genetic Algorithms in Engineering Systems: Innovations and Applications, GALESIA, vol. 414. 